\title{
Gradhiva
}

Revue d'anthropologie et d'histoire des arts

\section{Flûtes des hommes, chants des femmes}

Images et relations sonores chez les Kuikuro du Haut-Xingu

The flutes of men and the songs of women. Images and Acoustic Relations among the Kuikuro of the Upper Xingu

\section{Bruna Franchetto et Tommaso Montagnani}

\section{OpenEdition \\ Journals}

Édition électronique

URL: http://journals.openedition.org/gradhiva/2052

DOI : 10.4000/gradhiva.2052

ISSN : $1760-849 x$

Éditeur

Musée du quai Branly Jacques Chirac

Édition imprimée

Date de publication : 18 mai 2011

Pagination : 94-111

ISBN : 978-2-35744-042-5

ISSN : 0764-8928

Référence électronique

Bruna Franchetto et Tommaso Montagnani, «Flûtes des hommes, chants des femmes », Gradhiva [En ligne], 13 | 2011, mis en ligne le 18 mai 2014, consulté le 04 mai 2019. URL : http:// journals.openedition.org/gradhiva/2052 ; DOI : 10.4000/gradhiva.2052 
F.

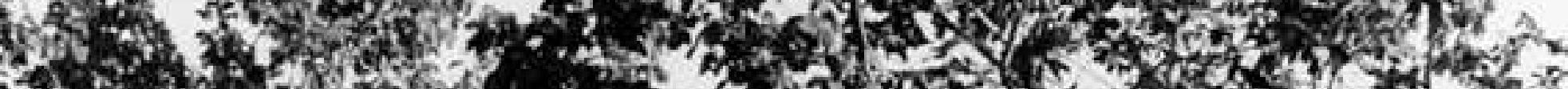

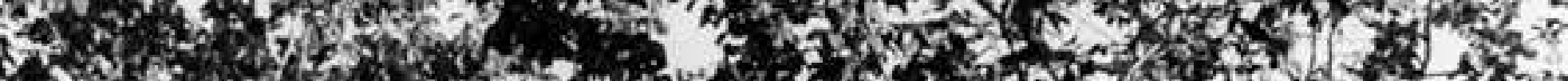

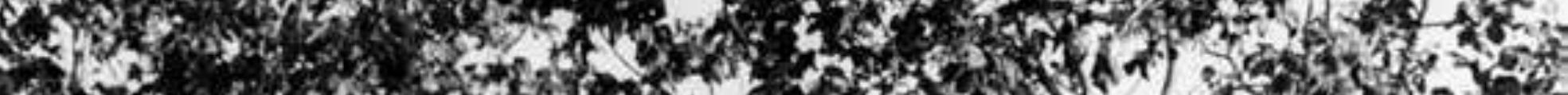

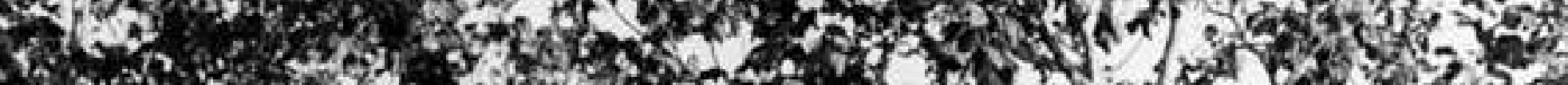

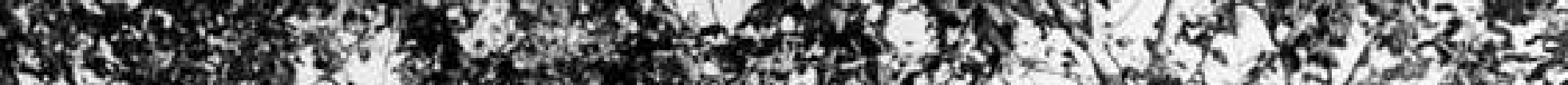

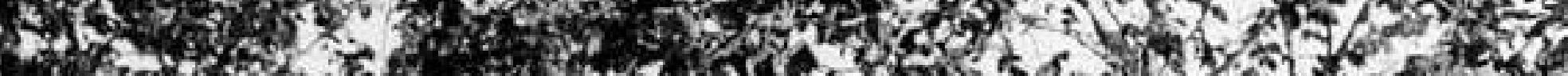

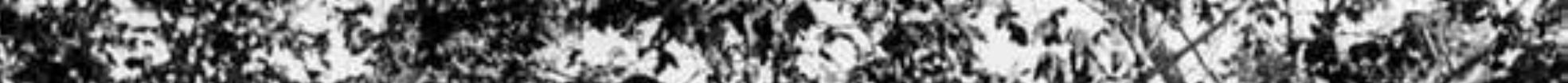

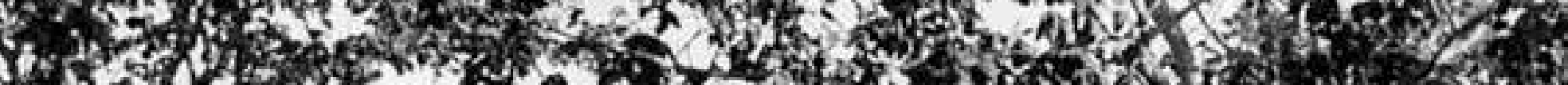

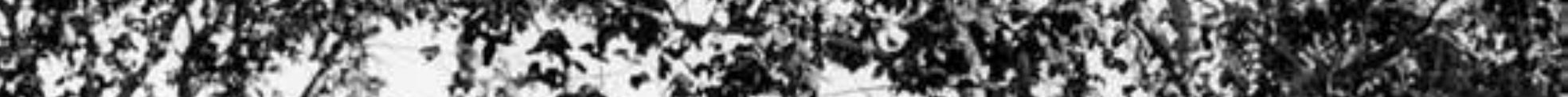

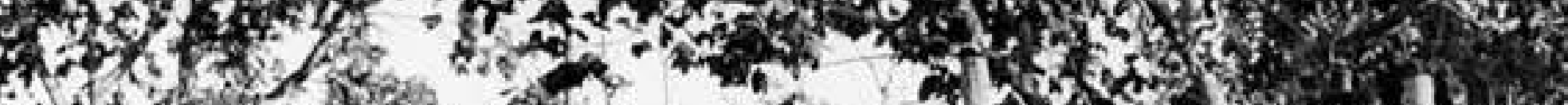

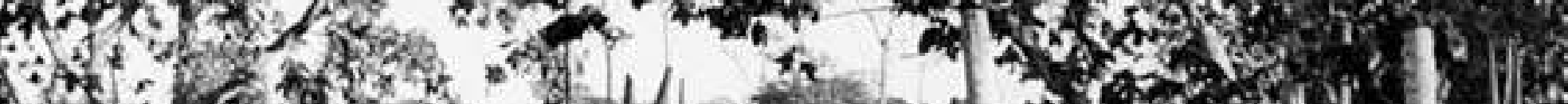

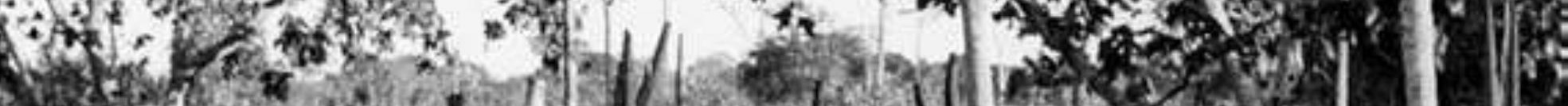
D.

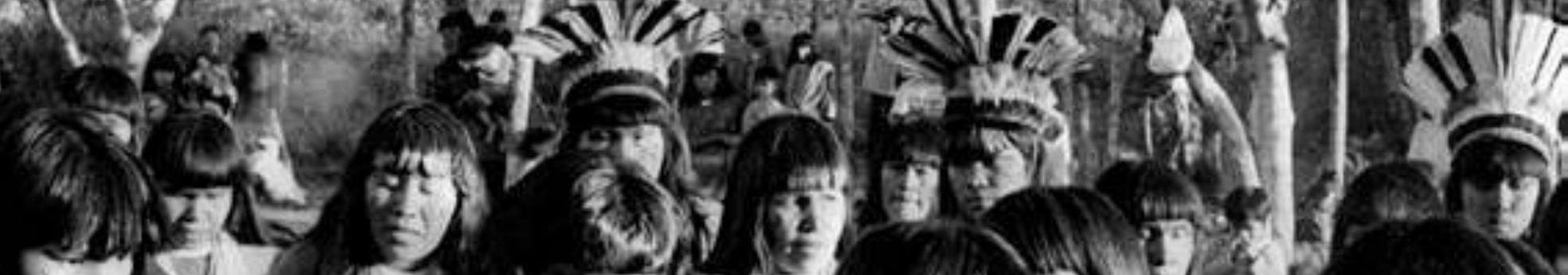

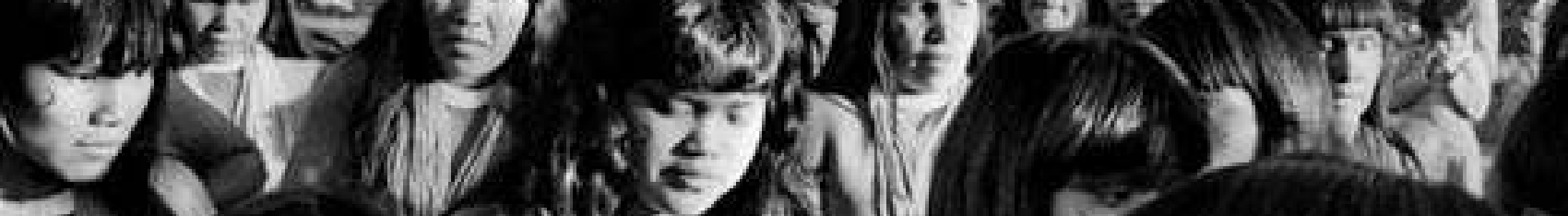
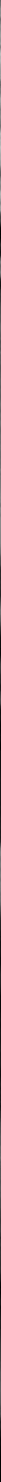


\section{Flûtes des hommes chants femmes}

\section{Images et relations sonores chez les Kuikuro du Haut-Xingu}

Bruna Franchetto
Tommaso Montagnani

Fig. 1 Les femmes kuikuro dansent dans le campement avant de faire leur entrée dans le village yawalapiti pendant un rituel tolo. Parque Indígena do Xingu, Brésil, 2005.

Photo Carlos Fausto.
Quelles sont les dynamiques créatrices qui surgissent de la relation entre musique et langage chez les Kuikuro du Haut-Xingu? Dans la musique du Haut-Xingu, des entités humaines et non humaines sont invoquées ou se manifestent sous forme de musique ou de parole. Le son des instruments de musique d'un côté et l'énonciation de noms propres de l'autre acquièrent une valeur et une fonction rituelle analogues dans deux différents contextes rituels, le rituel kagutu (rituel masculin) et le rituel tolo (rituel féminin). Nous allons essayer ici de décrire la façon dont certains éléments de deux contextes rituels séparés sont connectés par des analogies formelles et fonctionnelles. La connexion qui est formée par les spécialistes des deux rituels a pour effet de créer des entités qui dérivent d'une cumulation de subjectivités : la rencontre de mélodie et de texte accompagne et souligne la rencontre entre une entité surnaturelle et un personnage humain partageant une mélodie commune. La combinaison de la musique instrumentale et d'un texte poétique crée une nouvelle dimension rituelle dans laquelle des identités humaines et surnaturelles sont superposées dans une représentation chimérique de type sonore. L'union de la musique venant du répertoire masculin et du texte du répertoire de chants féminins donne lieu à la naissance d'une entité sonore qui est à la fois un humain, un esprit et un animal. Chacun des deux éléments (mélodie et texte) de l'objet musical complexe ici observé possède une existence et une fonction autonomes dans deux contextes rituels séparés : une mélodie est associée au nom d'un esprit dans le rituel masculin, et un nom propre à un personnage humain dans le rituel féminin. Dans ce dernier, 


\section{PIÈGES À VOIR, PIÈGES À PENSER}

l'énonciation du nom propre humain est accompagnée par le profil mélodique de l'esprit du rituel masculin, créant ainsi une référence directe à l'entité surnaturelle au moyen d'une superposition des référents. D'un autre côté, l'écoute de la seule mélodie dans le contexte du rituel masculin ne cesse d'évoquer une image sonore incomplète à laquelle l'identité humaine du chant féminin est mentalement associée par l'auditoire. Il apparaît évident qu'il est nécessaire de considérer les deux rituels comme formant un système de relations devant être observé dans sa totalité : le dialogue entre les sexes qui est tissé autour des deux pratiques rituelles se révèle donc indissociable de la relation entre humains et esprits représentée dans l'ensemble des connexions interrituelles autour de tolo et kagutu.

\section{Les maîtres des chants}

1. Six cents Kuikuro habitent dans six villages au sud-est de la Terre Indigène du Xingu en Amazonie méridionale. lls parlent une des variantes de la langue caribe du Haut-Xingu, un des deux rameaux méridionaux de la famille linguistique caribe (Meira et Franchetto 2005). Les données sur les chants kagutu présentées dans cet article ont été collectées par Bruna Franchetto au cours d'une recherche linguistique centrée sur les arts de la parole étalée de 1981 à 2006. Les données sur la musique kagutu ont été collectées par Tommaso Montagnani au cours de deux séjours sur le terrain (2008 et 2009) à Ipatse, le principal village kuikuro.

2. Le complexe rituel kagutu-tolo, auquel on devrait ajouter le rituel jamugikumalu, existe, avec des variations, dans tous les groups du Haut-Xingu (voir Piedade 2004 et Melo 2005, pour les Wauja, de langue arawak). La documentation linguistique de la langue caribe du Haut-Xingu a été réalisée dans le cadre d'un projet DOBES (20012006) ; depuis 2003, le groupe de recherche Documenta Kuikuro, coordonné par Bruna Franchetto et Carlos Fausto, au Museu Nacional de l'université fédérale de Rio de Janeiro, est en charge de la documentation des quinze rituels kuikuro ayant survécu jusqu'à aujourd'hui.

3. La forme possédée de tolo est celle où le radical nominal est précédé par le préfixe personnel et suivi par le suffixe de relation (ou possession], comme par exemple en utologu (1-tolo-REL), littéralement «mon tolo 》.
La question du genre se trouve au cœur des nombreuses correspondances qui existent entre deux des plus importants rituels chez les Kuikuro du Mato Grosso, au Brésil ${ }^{1}$ : celui des flûtes masculines kagutu d'une part et celui des chants féminins tolo ${ }^{2}$ de l'autre. Le rituel kagutu prévoit l'exécution d'un répertoire de dixneuf suites (au total environ six cents pièces) considérées comme appartenant aux itseke, les esprits, ces derniers étant désignés comme les «maîtres " (oto). Il s'agit d'une musique instrumentale, exécutée uniquement sur de longues flûtes en bois qui portent le même nom que le rituel. Les flûtistes qui y participent sont au nombre de trois : un soliste joue les thèmes des pièces et deux autres flûtistes exécutent une figure régulière qui a fonction d'accompagnement. Les deux accompagnateurs sont d'habitude des apprentis qui, souvent, ne connaissent pas le répertoire kagutu dans sa totalité. Le jeu de la flûte kagutu est réservé à une élite de maîtres flûtistes qui se recrute seulement parmi les hommes. La vision de l'instrument est même interdite aux femmes sous peine de viol collectif. Pendant le rituel tolo, un large corpus de chants considérés comme une transposition des musiques kagutu est exécuté par un chœur de femmes, et les hommes ne prennent alors pas part au rituel et restent très discrets, bien qu'il n'existe pas pour eux d'interdiction formelle d'y assister. Les femmes eginhoto (maîtres des chants) conduisent le chœur féminin. Les eginhoto (femmes et hommes) sont des spécialistes rituels jouissant d'un grand prestige dans la communauté qui leur vient d'un savoir rituel assimilé tout au long d'un processus d'apprentissage pouvant durer plusieurs années. Un flûtiste kagutu expérimenté est aussi appelé eginhoto.

Le mot tolo, dans la langue caribe du Haut-Xingu, signifie « oiseau » et, dans sa "forme possédée ${ }^{3}$ ", animal domestique (Franchetto 1997, 2001). Dans le contexte d'une relation sentimentale, les Kuikuro utilisent deux termes pour désigner très affectueusement les personnes qui leur sont chères : uhisü (mon frère cadet, mon petit frère) et utologu (mon tolo). Ces deux mots sont étroitement liés à des sentiments de protection et de plaisir inhérents à une liaison de dépendance réciproque en dehors des obligations du mariage. Le chant tolo qui suit est un exemple de cette connotation de utologu :

Katutolo uatinhi
Utologui(ni)
Tikinhü uatinhi
Utologui(ni)

Celui qui court devant tous

Est mon aimé

Celui qui court devant les gens des autres villages

Est mon aimé 


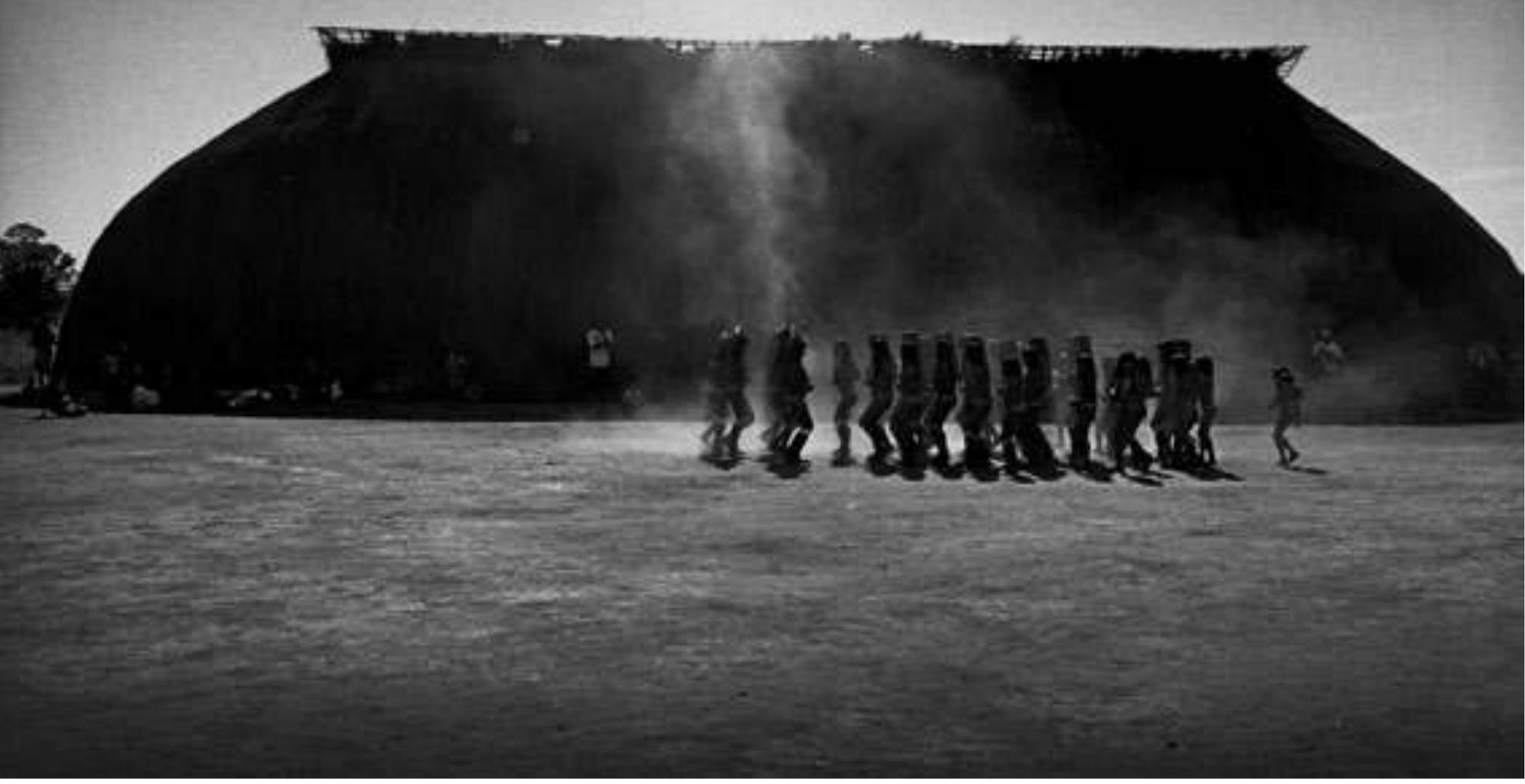

Fig. 2 Les femmes kuikuro dansent devant une maison pendant un rituel tolo dans le village yawalapiti. Parque Indígena do Xingu, Brésil, 2005. Photo Carlos Fausto.

En kuikuro, on utilise l'expression tolo-te-, (tolo-verbalisateur), «faire aller/ envoyer le tolo", un verbe transitif dont le patient est le destinataire : u-tolo-tepügü i-heke ( $1^{\text {re }}$ personne-tolo-verbalisateur-perfectif $3^{\mathrm{e}}$ personne-ergatif), «il a m'a chanté-tolo». Au moyen d'une structure d'enchâssement de citations, la chanteuse chante les tolo composés dans un passé éloigné par une autre femme qui les envoya comme message à son aimé; c'est cette femme compositeur qui, s'adressant à quelqu'un souvent identifié par son nom propre, parle à la première personne dans le texte («mon aimé, j'ai dit, je suis, je ne suis pas, etc. »).

Selon les Kuikuro, les deux répertoires sont basés sur les mêmes mélodies: les chants tolo ne seraient que les musiques des suites kagutu chantées avec un texte, un poème lyrique composé dans une des variantes de la langue caribe du Haut-Xingu. En réalité, nous verrons que la relation entre les deux répertoires musicaux, et par conséquent entre les deux rituels hommes/femmes, est plus complexe que cela et que le discours kuikuro sur la similitude entre les deux musiques est délibérément ambivalent. 


\section{PIÈGES À VOIR, PIÈGES À PENSER}

- $\circ$

4. L'analyse des procédés de répétition de la musique kagutu a été exposée dans Fausto, Franchetto et Montagnani (2011).

5. Cette tension entre autorité masculine et autonomie féminine anime aussi le rituel jamugikumalu (hyper-femmes chefs, en langue arawak), une autre «fête » des femmes, où des badinages, parfois violents, opposent les femmes et les hommes. En chantant les chants jamugikumalu, les femmes reproduisent leur transformation (etinki-, «s'inventer, à soimême »] mythique en itaõ kuegü, «hyper-femmes », femmes-esprits. Kuẽgü est un nom relationnel dont la racine $k u \tilde{u}$ est le concept de «hyper», un excès absolu. Le rituel reproduit le script du récit mythique $L a$ métamorphose ou le début des Hyper-femmes. Dans ce récit, les femmes abandonnées par leurs époux transformés en hyperpécaris après le rituel d'initiation des garçons (percement des oreilles] décident de danser avec les parures masculines et de chanter sur le kwakutu («maison des hommes ») pour devenir des êtres androgynes, puissants et dangereux. Comme disent les femmes pendant le jamugikumalu : itão kuegü higei kukuge («ici et maintenant nous sommes des hyper-femmes »). Les hyper-femmes du mythe se piquent le vagin avec des insectes jusqu'à faire grossir les lèvres et le clitoris. Ensuite, elles s'enfuient jusqu'aux frontières du monde pour y bâtir un village où, entre autres choses, elles jouent les flûtes kagutu (Franchetto 1996, 2003).

6. «Après que les hommes eurent reconquis les flûtes kagutu, enlevées par les hyper-femmes, les jamugikumalu, ce qui resta aux femmes c'était seulement chanter les kagutu. » (Tikugi, 2009) «Kagutu était aux femmes, mais les hommes le volèrent. C'est pour ça qu'elles ne peuvent plus jouer les kagutu. Après beaucoup de temps, elles commencèrent à chanter kagutu. Au début, peu à peu, après de plus en plus. Ça s'est passé parmi les anciens Kalapalo, les Akuku. » (Ipi, 2003)

\section{Variations cachées}

La relation entre les deux répertoires est-elle vraiment une relation de fidélité absolue comme l'affirment les Kuikuro? Dans le complexe système de relations entre kagutu et tolo, il existe de nombreux cas où la variation musicale est, pour ainsi dire, passée sous silence : elle est un trait crucial dont personne ne parle ni n'admet l'existence. La variation thématique n'est pas admise dans la musique kagutu. La musique de flûte sacrée est jouée de la même manière d'une occasion à l'autre : en comparant un certain nombre d'exécutions de plusieurs flûtistes, nous avons pu constater que, bien qu'il existe des différences dans le nombre de répétitions des phrases musicales, les mélodies ne subissent jamais de variation 4 . La comparaison entre les exécutions de la musique kagutu et celles des chants tolo présente en revanche des aspects plus problématiques, en particulier en ce qui concerne l'apparente contradiction entre le discours kuikuro sur la musique et les résultats de l'analyse des données en notre possession. Les femmes affirment s'en tenir au modèle masculin, traduire ou interpréter leurs propres chants sur les musiques de kagutu, mais il existe des différences assez importantes : le chant tolo s'écarte souvent des mélodies kagutu au point de créer un autre répertoire de mélodies. C'est à partir de là que les relations entre hommes et femmes se complexifient. Le système de correspondances formé par les rituels kagutu et tolo est caractérisé par une tension continuelle entre la différenciation et l'établissement de relations entre le monde masculin et le monde féminin. L'affirmation de l'autorité masculine d'un côté et la revendication de l'autonomie féminine de l'autre ne sont pas étrangères au fonctionnement de ce système rituel5. La dimension de l'autonomie féminine est pourtant cachée, et seule une analyse détaillée des analogies et des différences entre musique instrumentale masculine et musique vocale féminine permet de la mettre en évidence sous forme de «déviation " par rapport au modèle. On peut observer chez les femmes une sorte de négation ou d'understatement de la spécificité de leurs chants, qui sont beaucoup moins conformes aux mélodies du kagutu que ce que le discours kuikuro ne le laisse croire.

Selon le récit d'origine des suites kagutu, les itseke (esprits-animaux), invisibles aux êtres humains dans un état de conscience ordinaire, sont les «maîtres " (oto) et les créateurs des mélodies. Dans le mythe, Tãugi, le trickster créateur, chantait les suites dans son village avec les itseke. Chaque esprit, lorsqu'il commençait à chanter sa pièce, disait son nom, comme s'il s'agissait d'une sorte de présentation. Un bon nombre de pièces kagutu ont donc un esprit oto, qui donne aussi son nom à la pièce. Pendant leur exécution, la flûte soliste, chargée de l'exécution des thèmes, est censée reproduire ce nom sous forme de mélodie. Le nom de l'esprit maître n'est jamais chanté ou prononcé par une personne pendant le rituel, mais il est entendu dans le son de la flûte : selon les Kuikuro, la flûte "chante ", et peut donc prononcer le nom du maître. À l'intérieur de ces pièces se trouve donc un motif mélodicorythmique qui est en réalité le nom d'un esprit, comme nous le verrons plus tard.

Même si les commentaires des femmes reprennent souvent le thème du vol des flûtes kagutu par les hommes, dans une tonalité quelque peu nostalgique ${ }^{6}$, le vrai récit d'origine des chants tolo semble se trouver en contradiction avec le mythe d'origine des musiques kagutu : la paternité des mélodies n'y est pas attribuée aux esprits, mais à des femmes «historiques ".

Voici des extraits du récit raconté par Ajahi Kuikuro, l'une des principales chanteuses de tolo parmi les Kuikuro: 
Ça c'est tolo, il a commencé sur les marges du lac Tahununu.

Précisément à Agahahü, parmi les anciens Akuku

Nos anciens étaient chantés (tolote)

Elles chantaient pour Agahahü

"Où est celui dont nous sentons le manque?

Où est celui qui est attaché au lacet?

Là, loin, à Hagagikugu ”

Elles chantaient

Elles mentaient, elles se disputaient

Elles chantaient et disaient du mal d'elles-mêmes

Quand elles faisaient l'amour [avaient des histoires d'amour]

...

Elles ont fait (üi) le chant kagutu

C'est ça que nous appelons tolotepe, ce qui fut tolo

Elles dansaient

Et alors les hommes dirent «les femmes ne peuvent pas rester avec ces chants,

elles resteront sans eux"

Ce fut comme ça que les hommes s'emparèrent des chants des femmes

C'était les femmes qui composaient [cousaient] là-bas à Agahahü

C'était les femmes, aussi pour les chants plus importants, comme les sogoko

Les chants devinrent les musiques sogoko des kagutu

C'étaient elles qui composaient tout, les anciennes femmes Akuku

Là-bas à Agahahü, c'est là-bas que tout a commencé et c'est devenu notre fête.

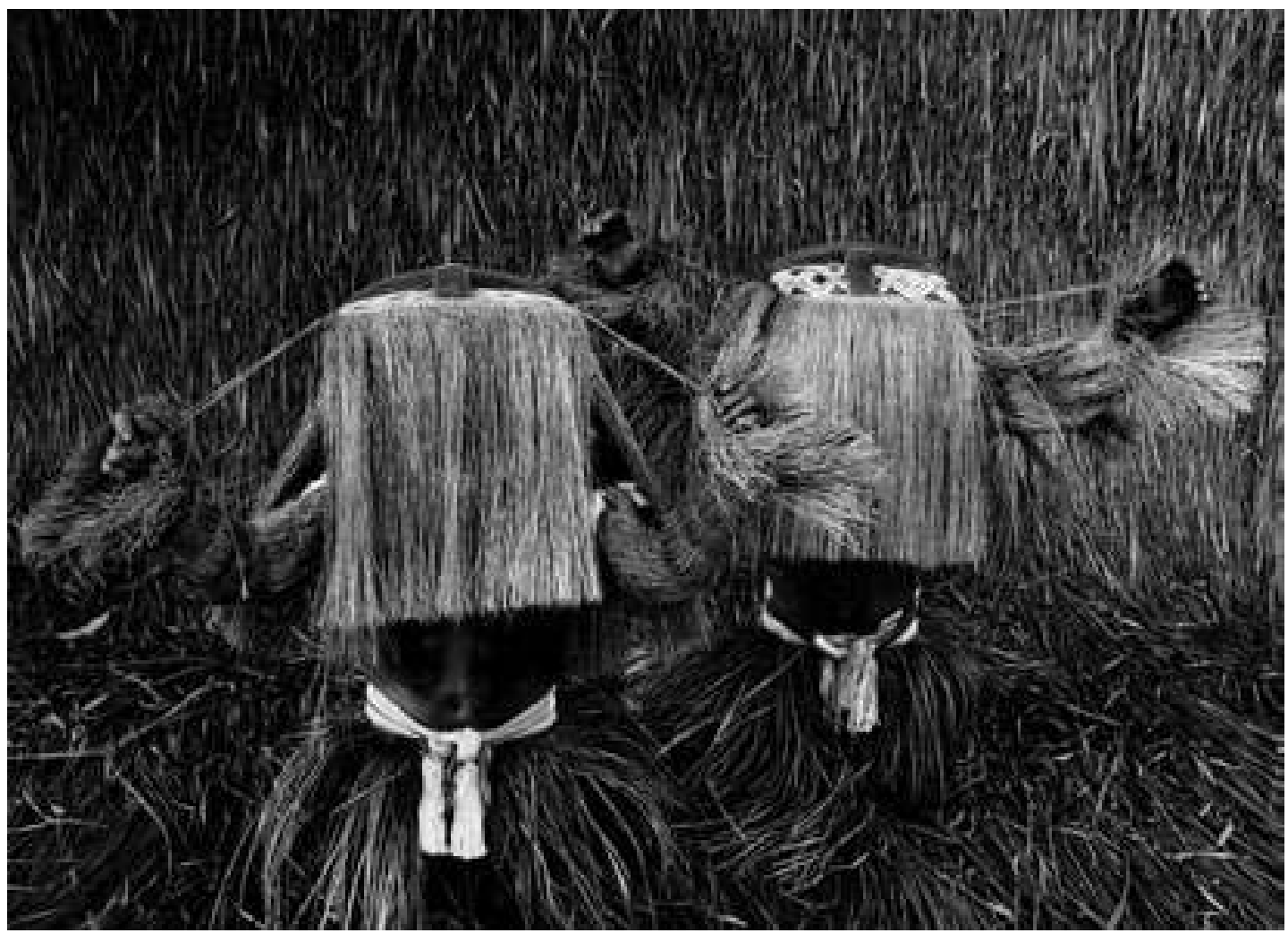

Fig. 3 Derniers préparatifs de la cérémonie Kuarup dans un village kuikuro. Les masques jakuikatu sont promenés dans le village en marque de respect pour l'esprit Jakuikatu qui doit être ménagé. Mato Grosso, Brésil, 2005. Photo Sebastião Salgado (c) Sebastião Salgado/Amazonas Images/Contact Press Images. 
Les Akuku ou Kalapalo sont les voisins des Kuikuro. Ils parlent une autre variante de la langue caribe du Haut-Xingu. Le fait que les Kalapalo soient cités dans ce récit est assez significatif : cela témoigne du caractère intertribal qui est propre à la fois à tolo et à kagutu (ainsi qu'à la majorité des rituels xinguaniens) et dont on retrouve la trace dans les récits originels.

Le récit d'Ajahi est particulièrement riche. Nous avons choisi de prêter une attention particulière à certaines expressions utilisées dans le récit, notamment à la racine üi (réalisée comme verbe transitif), dont le sens complexe et abstrait peut être traduit par "faire-transformer", "causer un changement radical d'état ». En langue kuikuro, l'utilisation de cette racine a été observée dans une grande variété de contextes ${ }^{7}$ : on üi les jeunes gens pendant la réclusion pubertaire (leur corps et leur ügühütu, leur façon d'être), l'enfant est üi dans le ventre de sa mère, on üi quelqu'un quand on le peint avec les motifs graphiques de la peinture corporelle. C'est une transformation créative et vivifiante ${ }^{8}$, et, dans le contexte qui nous intéresse, les femmes üi kagutu en chansons, parce qu'elles modifient la mélodie des esprits en chantant les tolo.

Nous nous trouvons donc face à un discours ambigu de la part des femmes. Elles affirment d'un côté, en accord avec les hommes, que ce qu'elles chantent, c'est la musique des esprits, et elles revendiquent de l'autre la paternité de la musique de kagutu, née aux bords du lac Tahununu, lieu réel dans le territoire originel des Caribes du Haut-Xingu, à l'est du fleuve Culuene, sur les marges duquel habitent les actuels Kuikuro et Kalapalo. Cette revendication devient particulièrement claire vers la fin du récit d'Ajahi. ("C'étaient elles qui composaient tout, les anciennes femmes akuku.»)

Pour une partie des pièces kagutu, les femmes ont mis des paroles et ont produit des chants. Ce sont des poèmes lyriques, images versifiées au parallélisme simple ou complexe, et aux citations enchâssées («on me fait dire (que) j’ai dit à mon amour... "), des scènes ou des scénarios minimalistes qui suggèrent une histoire d'amants, dramatique ou légère :

Tuãka kete

Uhisü kilü uheke

Utalitsügü kutsonkgitomi

Umüngitsügü kutsonkgitom
Allons prendre un bain

J'ai dit à mon petit frère

Lave-moi et enlève-moi un peu de mon parfum de copaïba

Lave-moi et enlève-moi un peu de mon roucou.

Ce qui frappe en comparant le rituel masculin et les chants féminins est la distance entre le caractère sacré (tainpane) des pièces et des exécutionws kagutu, leur " tristesse " («les flûtes pleurent » selon les Kuikuro) et l'ironie, l'éclat joyeux des messages profanes des tolo. Ajahi dit : "Nous nous disputons autour des nos amants, le mensonge est toujours dans les interlignes, nous ne valons rien. " Elle semble ici adopter, dans une posture d'autodénigrement, le point de vue masculin : les femmes sont dites aũgene oto, «maîtres du mensonge et de la rumeur ». Mais le rire d'Ajahi lorsqu'elle prononce cette phrase indique qu'on peut la comprendre, par un jeu d'antiphrase courant chez les Kuikuro, dans le sens contraire : «Nous avons de la valeur. " Les femmes dirigent d'ailleurs la politique des coulisses domestiques qui se répercute avec toute sa force dans la politique des hommes à l'intérieur du village comme entre les villages. Reprenons en détail ce que dit Ajahi à propos de ce «mensonge ", avant de discuter le sens complexe du terme kuikuro : 
Tita leha itsolotegagü

C'était là [à Agahahütü] qu'elles chantaient-disaient [Tolo-te-, « faire aller les chants tolo, chanter-dire »]

Augũtako hegei

Elles mentaient

Otolotehesundako hegei

Elles chantaient-disaient du mal d'elles-mêmes

Ai gehale tü isajotunalüko tüha

Quand elles avaient des amants

Ülepe Tolotengalü leha ihekeni

Elles chantaient-disaient

Talokito ekutsügü uagindua tsüha

Elles n'avaient aucune valeur, comme moi

Ah ah ah... [Ajahi rit]

Üleingo gele taka igei nalü

Ainsi ça continue à être

«Tapogingotse Atujügüni Atujügüni [Ajahi chante un tolo]

«Il est joli, Atujügü

Atujügüni Atujügüni

Atujügü Atujügü

Angiha[la]kanga isagingoni

Il y a quelqu'un qui lui ressemble

Asuti tendühükingo ihinhano"

C'est le frère aîné qui ressemble à un crapaud brûlé ”

Tatohoi hegei

Elles disaient

Téhe Katujügü hekite

Comme Katujügü était beau

Ihi... engütsügü hüle ihisü hüle ekisei an

Il était le frère cadet

Hesinhüi hüle

Du mauvais

Üngele heke hegei tagü asuti tendühügü hungu tatohoi

De celui-là, elles disaient qu'il ressemblait à un crapaud brûlé

Asuti agilütsüha itohonga ülepe...

Comme quand on jette un crapaud dans le feu

Üle agage leha ihata ihekeni

C'était comme ça qu'elles disaient

Ngihũgu tisihũgu hekeha un

Autour de nos ancêtres

Tatute leha

Autour de tous

Kagutu igisüi leha tüilü leha

Elles firent le chant de kagutu

Igeiha Tolotepe titsingatohoi

C'est ça que nous appelons Tolotepe, ce qui fut tolo [Tolo-te-pe, tolo-faire.aller-Passé]

Ige higei ago notagüi higei Tolo igisüiha ülepe

C'est ça qu'elles sont en train de chanter maintenant, les chants tolo. 
PIÈGES À VOIR, PIÈGES À PENSER 
La racine aũ (dont la traduction « mentir/mensonge " est approximative, partielle et peut-être équivoque) exprime les infinies possibilités créatrices du langage : grâce à cette capacité d'invention, qui est propre à l'activité langagière humaine, la parole possède le pouvoir de créer d'autres mondes possibles qui, dans la mythologie kuikuro, peuvent devenir réels et attirer les humains à leur intérieur (Franchetto $19^{8} 6^{9}$ ). On pourrait ainsi faire dire aux femmes «nous faisons semblant de chanter les musiques kagutu, mais en réalité, nous créons, nous transformons la mélodie originelle jusqu'à la bouleverser; c'est un mensonge et un divertissement ". C'est une ironie malicieuse et consciente qui accompagne le refus de l'« imitation " en tant que subordination à un modèle qu'il faudrait reproduire fidèlement.

Le mensonge et surtout le non-dit tiennent un rôle primordial dans les dynamiques autour du système rituel kagutu/tolo. En transformant les musiques kagutu, les femmes accomplissent un acte de transgression, dans le mythe et dans la fête. Il faut introduire ici une distinction importante entre ce qui est déclaré, reconnu par la communauté, et ce qui est passé sous silence. Ce qui est passé sous silence ne transparaît que dans l'analyse musicologique et dans les récits d'origine des chants tolo, racontés par les femmes quand les hommes sont absents ou très peu présents.

Dans but de décrire le fonctionnement des dynamiques qui s'instaurent autour de la relation hommes/femmes dans le système rituel kagutu/tolo, nous introduisons ici les concepts de relation symétrique et complémentaire, selon les modèles d'analyse proposés par Bateson (1958) et Severi et Houseman (2009) pour la société iatmul de Nouvelle-Guinée :

«En général, on appellera [...] symétrique toute "différenciation progressive" qui se réalise par une compétition fondée sur l'exhibition de la même réponse relationnelle et complémentaire, celle qui se fonde sur deux attitudes relationnelles différentes ${ }^{10}$. "

Lorsque, par exemple, un individu affiche un comportement autoritaire et qu'un autre répond par une attitude de soumission, nous nous trouvons face à l'instauration progressive d'une relation complémentaire. La relation est symétrique lorsque les deux individus adoptent le même comportement : la réponse à un comportement autoritaire comme l'obligation de se tenir au répertoire imposé par les esprits (c'est le cas que nous observons ici) serait l'exécution d'un autre répertoire «antagoniste ». Le cas kuikuro est particulièrement complexe non seulement parce que nous avons trois pôles de la relation (hommes, femmes et esprits) mais aussi parce que le comportement des femmes et leur positionnement vis-à-vis de l'autorité masculine et des esprits sont ambigus. Un autre facteur qui contribue à rendre complexe l'analyse des relations est le temps des actions rituelles : les deux rituels ne sont pas exécutés en même temps. Plusieurs mois peuvent séparer l'exécution d'un kagutu de celle d'un tolo. Cependant, pour les raisons que nous avons exposées en ouverture, nous pensons qu'il est nécessaire de lire ces actions et les enjeux des deux rituels de manière conjointe. Nous avons ici fait le choix d'étendre l'analyse des relations rituelles à deux événements séparés, qui sont néanmoins connectés par des analogies musicales et par des représentations des relations sociales entre hommes et femmes.

Si l'on adopte le point de vue « officiel » kuikuro (contredit, il est bon de le rappeler, par les récits «non officiels » d'Ajahi) et que l'on considère une antécédence temporelle de kagutu par rapport à tolo, on assiste à un mécanisme de transfert : l'autorité des esprits, qui est perçue par les hommes pendant le kagutu sous forme d'une menace de maladie ou de mort en cas de mauvaise exécution, est ensuite exercée sur les femmes par les hommes eux-mêmes. Les hommes occupent donc
- $\odot$

9. Basso (1987) a décrit chez les Kalapalo la centralité du concept exprimé par auN, qu'elle a traduit par deceit, deceitful. Le nom de l'itseke Taugi, le créateur, est dérivé de la forme participiale du verbe auN-guN-(auN-verbalisateur), «celui qui est dans la condition de auN, qui ment/crée». Dans les récits mythiques kuikuro, Tãugi et son frère jumeau Aulukuma créent à partir du langage mensonger, par le simple acte de donner de faux noms aux choses. Tãugi corrige ensuite les faux noms en établissant pour chaque chose le «vrai » nom, ce qui réduit la création originale à une dimension appauvrie et la rend possible, réelle, adéquate.

10. Houseman et Severi (2009 : 35). 
une place intermédiaire, dans laquelle ils subissent une autorité d'un côté et l'exercent de l'autre. Le rôle des femmes est encore plus difficile à définir et, pour cela, il est nécessaire de se placer du point de vue d'Ajahi, décrit dans les récits que nous avons présentés. Les femmes acceptent initialement l'autorité de la version kagutu et chantent la même mélodie que les hommes : on pourrait donc penser à une relation complémentaire dans laquelle l'autorité est exercée en même temps par les hommes et les esprits et subie en même temps par les femmes et les hommes. Progressivement, cette relation se transforme tout au long du rituel tolo, car les femmes commencent à chanter une mélodie différente et se placent ainsi dans une position antagoniste par rapport aux hommes et aux esprits : les chants tolo s'écartent des mélodies "sacrées " après en avoir exposé les thèmes initiaux, comme nous allons le montrer. Il s'instaure donc progressivement une relation symétrique, à la fois entre hommes et femmes et, surtout, entre femmes et esprits. Rituel et mythe se retrouvent sur le même plan lorsque les femmes instaurent une relation compétitive symétrique avec les itseke en transformant leurs mélodies et en se projetant ainsi dans la position des hyper-femmes du mythe en train de créer leurs propres mélodies. La singularité de ce modèle de relations est due au fait que nous avons trois pôles dont deux adoptent des comportements variables et/ou contradictoires. Les hommes instaurent deux relations de type complémentaire et leur position à l'intérieur de la relation change à chaque fois, puisqu'ils subissent l'autorité des esprits dans la première relation complémentaire et qu'ils l'exercent ensuite dans la seconde sur les femmes. Les femmes acceptent l'autorité masculine en respectant le tabou qui leur interdit de voir les flûtes et en reproduisant en partie les mélodies des itseke, mais elles défient les deux autres pôles de la relation : grâce à leur autonomie créatrice, elles modifient la musique des itseke et donnent naissance à de nouvelles parties musicales. Cette autonomie créatrice est enracinée dans le langage poétique des tolo, qui fonctionne comme moteur principal du développement du matériel musical venant de kagutu.

\section{Flûte des hommes et voix des femmes : esprits et amants}

On montrera ici la différence qui existe entre la pièce utigi de la suite kagutu appelée Tolo Imitoho et la version vocale tolo de la même pièce. Le but est d'illustrer ce qui a été exposé dans les pages précédentes : la version tolo des pièces kagutu n'est pas vraiment fidèle au modèle. On constatera en effet que le seul matériel en commun est en réalité le thème, i.e. la courte phrase mélodique qui commence la pièce (l'incipit). Nous l'appellerons "thème/identité ${ }^{11}$ " car il comprend toujours un fragment où l'on énonce le nom propre d'une entité non humaine (dans kagutu) ou humaine (dans tolo). Ce nom est aussi celui de la

11. Voir aussi l'idée de «devise musicale » chez Rouget (1980) et la «signature thématique » de Beaudet (1997). Ce qui caractérise le thème/identité et qui justifie l'adoption d'une expression différente de celles utilisées par Rouget et Beaudet est le fait que le thème/identité est étroitement lié à l'énonciation d'un nom. Ce que les Kuikuro entendent n'est donc pas simplement un rythme ou une mélodie, mais un nom propre. pièce musicale. Néanmoins, même ce thème/identité est légèrement différent dans la version vocale. Quant au reste du matériel mélodique de la version tolo, il n'a aucun rapport direct avec la version kagutu, en dépit du fait que, selon les Kuikuro, il s'agirait de la même mélodie. On observe en réalité un développement considérable du matériel mélodique dans la version féminine, qui est à la fois plus long et plus complexe dans sa structure comme rythmiquement.

Dans les deux transcriptions qui suivent, le thème/identité est noté en bleu. Dans la version kagutu, le fragment mélodique où le nom de l'esprit est entendu par les Kuikuro dans le son de la flûte correspond au moment où, dans le mythe, l'esprit prononce son propre nom (Utigi, un esprit/poisson). 


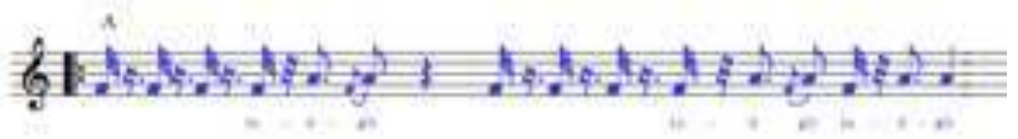

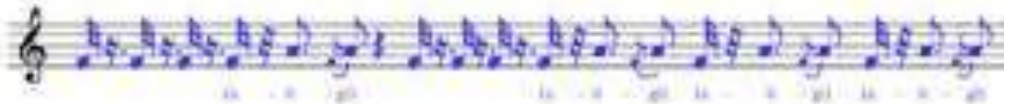
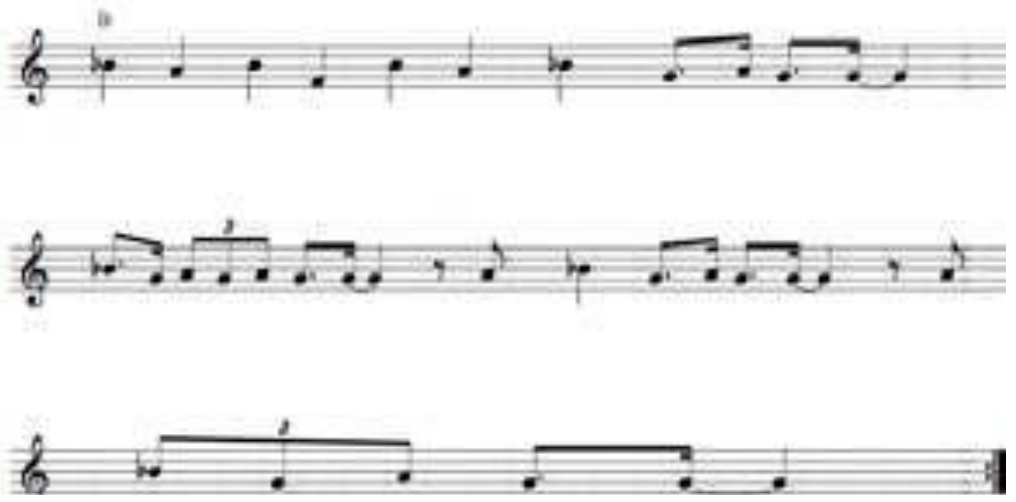

Pièce Utigi de la suite Tolo Imitoho, rituel des flûtes kagutu, exécuté par les hommes.

Voici donc la version vocale féminine, Uguta Hutaki.
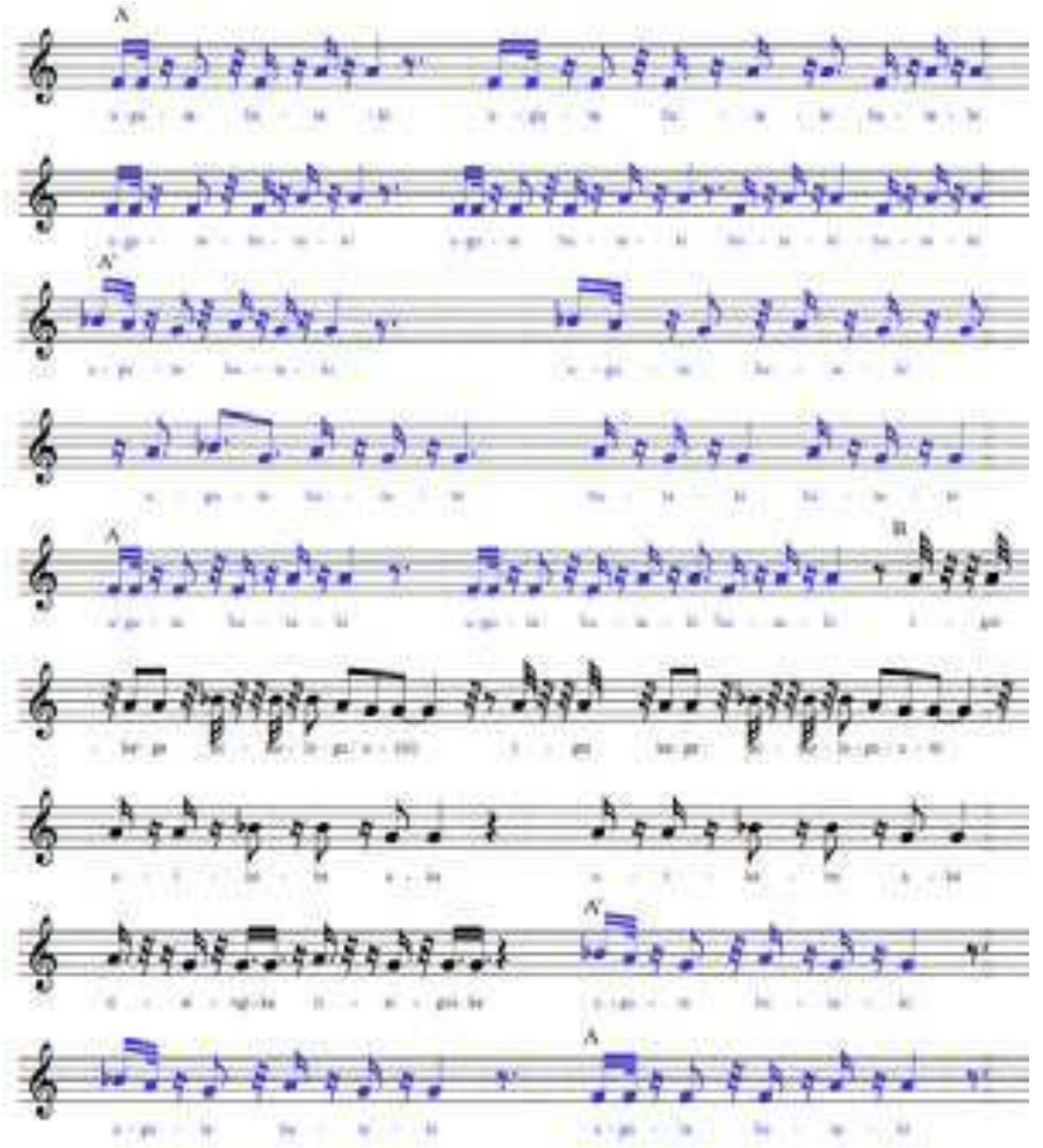

Uguta Hutaki, pièce de tolo, chantée par les femmes. 

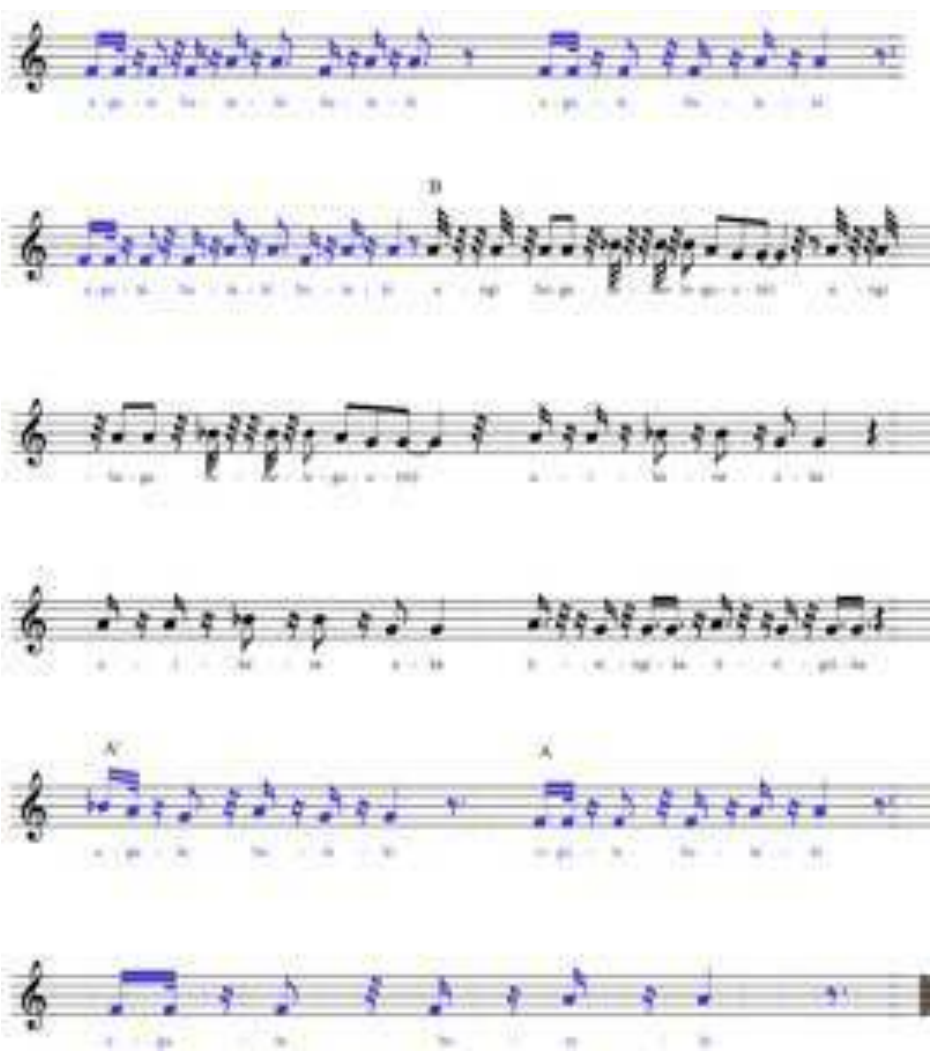

Le « texte » transcrit et traduit de ce chant tolo est le suivant :

Uguta Hutaki

Ingikege tsitsologu(ni)

notre petit oiseau [notre animal familier]

Uguta Hutaki

Angihuga tsitsologu(ni)

Uikene ake

Tisingike

Tisingike
Bouche-de-Cascudo

Viens voir

Bouche-de-Cascudo

Notre petit oiseau est angihuga ${ }^{12}$

Avec ma sœur plus jeune

Viens nous voir

Viens nous voir

Uguta Hutaki est un nom propre et il s'agit du personnage principal de la petite histoire racontée dans le chant. Ce qui était un nom d'esprit (Utigi) dans la version instrumentale kagutu est devenu le nom d'un personnage, l'amant appelé par la femme dans la version vocale tolo. Le fragment mélodique commun à l'esprit Utigi et au personnage Uguta Hutaki est encore une fois noté en bleu sur la portée, afin de montrer la transformation musicale de cette prétendue identité mélodique. Dans le passage d'«Utigi » (trois syllabes) à " Uguta Hutaki » (six syllabes), on observe une complexification du rythme qui est due à la fois à l'adaptation de la mélodie au nouveau nom et au type de phrasé qui caractérise le chant tolo. L'intervalle en revanche reste le même (une tierce majeure), ainsi que la séquence fa-la-la, ce qui garantit une continuité entre les deux versions. Le thème/identité d'Uguta Hutaki subit en outre une variation interne à la pièce tolo : ce phénomène peut être observé sur les troisième et quatrième portées. Il s'agit d'un type de variation qui est propre au tolo. Dans la pièce kagutu Utigi passereaux (Cotingidæ). tandis que dans le chant tolo correspondant, le nom du personnage est chanté 
sur une seconde mélodie, outre la mélodie principale dérivée du kagutu. Cette seconde mélodie est une variation sur la première et elle en garde le profil rythmique. Il est aussi important de signaler que si Uguta Hutaki est un nom propre de personne et Utigi le nom d'un esprit, tous deux sont aussi des noms de poissons ${ }^{13}$.

Les parties notées en noir sont propres à la version tolo. Elles n'existent pas dans la pièce kagutu, et sont donc une invention féminine, une mélodie nouvelle qui naît du développement musical produit par la présence d'un texte. Les paroles des chants tolo ont une influence directe sur la partie musicale, déterminant à la fois la transformation du thème/identité et la création de nouvelles mélodies. La structure de la pièce subit une modification, encore une fois dans le sens d'une complexification des valeurs rythmiques due à l'adaptation des motifs aux syllabes du texte (ce qui donne lieu à une augmentation du nombre de notes) et d'un développement de la structure-modèle, dû aux nouvelles mélodies introduites dans le tolo, à leur tour générées par l'introduction de la partie textuelle. On observe donc une séquence du type $\mathrm{A}, \mathrm{B}$ dans la version kagutu, tandis que la séquence des phrases musicales de la version tolo est $A, A^{\prime}, A, B, A^{\prime}, A, B, A^{\prime}, A$.

Examinons maintenant un second exemple tiré de la pièce kagutu appelée Tungingi et du chant tolo Taga/Kokogo. Cette fois-ci, nous avons, dans les deux versions, transcrit seulement la partie "thème/identité", et nous avons noté en rouge les fragments correspondant à l'énonciation d'un nom propre. On observe ainsi encore une fois que le même fragment mélodique se réfère selon le genre musical à des entités différentes. Alors que dans la version kagutu il renvoie à l'itseke appelé Tungingi (serpent aquatique), il est utilisé dans la version tolo pour prononcer et chanter le nom du personnage de l'histoire, Taga/Kokogo ${ }^{14}$. Dans le passage de kagutu à tolo, la mélodie subit une légère modification rythmique pendant sa première exposition : l'esprit de la version instrumentale (Tungingi) porte un nom à trois syllabes, tandis que le nom Taga, dans la version chantée, n'en compte que deux. Les deux croches (si-si) correspondant aux syllabes -ngi -ngi sont donc remplacées par une simple noire (si), correspondant à la syllabe -ga dans le texte tolo.

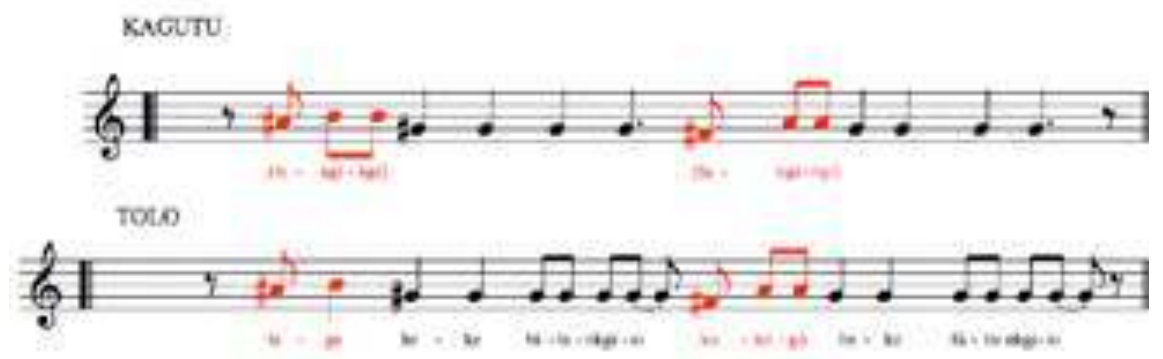

Les « paroles » du chant tolo sont les suivantes :

Taga heke tütankgisi anügü

Kokogo heke tütankgisi anügü

Tütankgisi huãke anügü

Ukilü hegei

Tühetunetiha anügü

Ijogu heke huãke

Taga heke huãke

Kokogo heke huãke $\circ \circ$

13. Le nom brésilien du poisson utigi est cachorra [《chienne», Cynodon gibbus, Characidæ] et celui d'uguta est cascudo [Glyptoperichthys xinguensis].

14. Les Xinguaniens cumulent plusieurs noms propres au cours de leur vie : le personnage du tolo est ici appelé par deux de ses noms. Taga et Kokogo sont aussi les noms de deux oiseaux : curicaca (Theristicus candatus] et corbeau [Corvus brachyrhynchos]. 
Comment le fragment mélodique kagutu est-il "fait/transformé » (üi) par les femmes dans la version tolo? Cette transformation concerne d'abord les protagonistes, et opère à plusieurs niveaux : non seulement un itseke (esprit) devient kuge (un être humain), mais un serpent aquatique devient deux oiseaux.

\begin{tabular}{|c|c|}
\hline MASCULIN & FÉMININ \\
\hline KAGUTU & TOLO \\
Tungingi (serpent aquatique) & Taga (oiseau curicaca) \\
ESPRIT & Kokogo (corbeau) \\
& ÉTRE HUMAIN \\
\hline
\end{tabular}

La musique accompagne ces deux types de transformations : comme on l'a déjà montré, le rythme s'adapte au nombre de syllabes. Ce n'est pourtant pas la seule métamorphose musicale. Prenons d'abord en considération la transcription de la version kagutu: le fragment mélodique que nous avons appelé thème/identité est ici exposé deux fois de suite avec une variante. Sa structure rythmique est la même dans les deux cas (trois croches), mais l'intervalle et la hauteur du fragment changent. Dans la première exposition, le thème/identité est formé par un intervalle d'un demi-ton, tandis que dans la seconde exposition, il s'agit d'un intervalle plus ample, une tierce mineure. En outre, cette seconde exposition est exécutée dans une tessiture plus grave que la première. La relation entre les deux fragments est une relation que nous appellerons de "synonymie musicale". Ces sont deux fragments différents qui sont mis en relation entre eux par le nom de l'esprit qui leur est associé. Ce nom, il est utile de le rappeler une nouvelle fois, n'est pas chanté ou prononcé par une personne, il est entendu par l'auditoire dans le son de la flûte. On retrouve ici certains des procédés génératifs qui sont à la base des arts de la parole, comme le principe du otohongo (" autre-même " ou « même-autre "). Ce dispositif métalinguistique est utilisé par les Kuikuro pour définir la duplicité qui produit dérivation, synonymie et répétition parallélistique, non seulement dans les récits, les formules chamaniques, les discours rituels, mais aussi dans la parole quotidienne, informelle (Franchetto 2003; Fausto, Franchetto et Montagnani 2011). Les Kuikuro disent que toute synonymie est une relation otohongo et qu' «il y a toujours un autre nom, une autre façon de dire la même chose ». Les fragments mélodiques (il est important de rappeler que nous parlons ici d'une musique instrumentale) sont mis en relation entre eux moins par les notes qui les forment que par l'identité à laquelle ils se réfèrent.

Voyons maintenant le cas de la version tolo de la même pièce. Dans ce cas, la relation que nous avons appelée synonymie musicale entre les deux fragments est soulignée par le texte : Taga et Kokogo sont deux noms différents, mais ils appartiennent à la même personne. 
Ce que la musique semble donc suggérer tout au long des multiples processus de transformation de kagutu à tolo est que non seulement Taga est l'autre nom de Kokogo, mais aussi que ces deux noms sont les noms humains de l'esprit Tungingi. Le signifié (l'itseke en question) change de signifiant selon le contexte rituel dans lequel il est évoqué. En revanche, si l'on prend à la lettre le signifié de chacun des trois noms utilisés dans les deux versions de la pièce (Tungingi, Taga, Kokogo), nous constatons qu'une identité surnaturelle, un itseke, est à la fois un serpent, un corbeau et un oiseau curicaca, selon le point de vue rituel d'où on l'observe. Une chimère sonore donc, ne prenant forme que dans la mise en relation de deux répertoires qui, tout en appartenant à deux rituels différents, contiennent les éléments mélodiques permettant d'opérer les connexions nécessaires à la manifestation de la chimère.

La pièce que nous venons d'analyser montre que le principe de synonymie/ parallélisme (otohongo) est donc utilisé non seulement à l'intérieur d'une pièce, mais aussi entre deux pièces appartenant à deux répertoires (et deux rituels) différents, établissant ainsi des correspondances interrituelles au moyen d'un procédé stylistique qui s'adapte aussi bien au texte qu'à la musique.

Dans le texte tolo qui suit, on montre une autre façon de tracer des parallélismes et des correspondances entre le personnage humain du tolo et l'esprit maître de la pièce kagutu. Ce chant est la version tolo de la pièce kagutu de l'esprit Tsügi :

\section{Uge esei}

Ahasahüngü talugei

Uge esei

Jamatuahüngü talugei

Uge esei

Ahintsahüngü talugei

Uge esei
C'est moi

Je ne suis pas le monstre Ahasa

C'est moi

Je ne suis pas Jamatua

C'est moi

Je ne suis pas un présage funeste

C'est moi

L'exégèse de ce court "texte", donnée par le flûtiste Tütekuegü Kuikuro ${ }^{15}$ et par sa cousine Kanu, est la suivante : un homme s'est introduit furtivement dans la maison de son amante, qui s'est effrayée de cette apparition nocturne. L'amant alors la rassure, en lui disant «c'est moi » (uge esei). Or, la mélodie de cette expression est le même fragment qui, dans la version kagutu, correspond au nom de l'esprit, Tsügi. Comme le mythe de la musique kagutu le raconte, l'esprit chante son nom en ouverture de la pièce afin de se présenter aux autres itseke. Qui est donc réellement en train de dire «c'est moi » dans le chant tolo? L'amant de l'histoire du kagutu et l'esprit Tsügi accomplissent le même geste, se présenter, en utilisant la même mélodie. La scène racontée dans le mythe et reproduite en musique pendant le rituel kagutu (Tsügi se présente aux autres esprits) est transposée dans le chant kagutu sous la forme d'une histoire d'amants. Le fragment mélodique commun aux deux versions indique que malgré le changement de contexte et la transformation du cadre, la présence de l'esprit reste, et que l'itseke Tsügi continue d'annoncer son arrivée même sous la forme d'un humain.

\footnotetext{
-

15. Tütekuegü est un flûtiste kagutu qui connaît bien aussi les textes des chants tolo. Tous les flûtistes ne connaissent pas ces chants, mais certains sont en mesure de les chanter et de les commenter avec leurs femmes dans l'espace domestique.
} 
-

16. Outre le discours kuikuro, nos analyses sont fondées sur la comparaison de plusieurs exécutions d'une même pièce. La version de Tütekuegü présentée dans cet article a été comparée avec celle de Jakalu, Kamangagu et Tupà, les autres flûtistes présents dans le village au moment de notre enquête de terrain.

\section{Femmes-esprits}

Les chants tolo amènent l'esprit maître de la pièce kagutu dans un nouveau contexte : le paysage musical dans lequel l'esprit est inséré est transformé par les femmes qui créent de nouvelles mélodies, modifient les structures de la pièce et nomment l'esprit par les noms de leurs amants. Le thème/identité est l'élément clé de la relation entre les deux répertoires et les deux rituels. Il véhicule la présence de l'esprit là où elle ne serait pas prévue, dans des chants profanes. Dans ces chants, la mélodie affirme ce qui est nié ou omis par le texte, à savoir que l'esprit est bien là malgré le sujet traité par les tolo et l'histoire racontée. Seulement, la relation que les femmes établissent avec les esprits est en quelque sorte opposée à celle que, dans la musique kagutu, les hommes établissent avec ces mêmes entités. La relation hommes-esprits est de type "complémentaire " : les maîtres flûtistes se soumettent à l'autorité des esprits. Ils observent strictement la version des itseke ${ }^{16}$, par crainte de leur vengeance. La relation femmes-esprits est de type symétrique, car elles exercent leur créativité dans les chants tolo. Tout en conservant la présence de l'esprit (le thème/identité), elles modifient les mélodies kagutu jusqu'à créer un répertoire nouveau et amener, au moyen des textes, les esprits dans l'univers de la parole féminine. En chantant tolo ou jamugikumalu, les femmes caribes du Haut-Xingu deviennent les Hyper-Femmes du mythe, des femmes-esprit, comme elles le disent elles-mêmes.

Programa de Pós-Graduação em Antropologia Social, Museu Nacional, Universidade Federal do Rio de Janeiro bfranchetto@yahoo.com.br EHESS, Laboratoire d'anthropologie sociale - musée du quai Branly tommmont@libero.it 


\section{Bibliographie}

\section{BAsso, Ellen}

1987 In Favour of Deceit. Tucson, University of Arizona Press.

\section{BATESON, Gregory}

1958 Naven. Stanford, Stanford University Press.

\section{BEAUdet, Jean-Michel}

1997 Souffles d'Amazonie. Nanterre, Société d'ethnologie.

\section{Fausto, Carlos, Franchetto, Bruna} et Montagnani, Tommaso

2011 «Les formes de la mémoire. Art verbal et musique chez les Kuikuro du Haut-Xingu (Brésil) », L'Homme 197 : 41-?0.

\section{FrancheTto, Bruna}

1986 Falar Kuikuru: estudo etnolinguístico de um grupo caribe do Alto Xingu.

Rio de Janeiro, Museu Nacional-UFRJ, thèse de doctorat.

\author{
FranchetTo, Bruna \\ 1996 «Mulheres entre os Kuikuro », \\ Revista de Estudos Feministas 4[1] : 35-54. \\ [Traduction anglaise par Cristopher \\ Peterson, «Women Among the Kuikúro », \\ ibid. : 225-239.] \\ 1997 « Tolo Kuikuro: diga cantando o que não \\ pode ser dito falando », Invenção do Brasil. \\ Revista do Museu Aberto do Descobrimento \\ $1: 57-64$. \\ 2001, «Ele é dos outros: gêneros de fala \\ cantada entre os Kuikuro do Alto Xingu », \\ in Cláudia Neiva do Mattos, Elizabeth \\ Travassos et Fernanda Teixera de Medeiros \\ (éd.), Ao encontro da palavra cantada: poesia, \\ música e voz. Rio de Janeiro, ?Letras-CNPq : \\ 40-52. \\ 2003 « L'autre du même : parallélisme et \\ grammaire dans l'art verbal des récits \\ kuikuro (caribe du Haut-Xingu, Brésil) », \\ Amerindia 28 : 213-248.
}

\author{
Houseman, Michael et Severi, Carlo \\ 2009 Naven ou le donner à voir. \\ Essai d'interprétation de l'action rituelle. \\ Paris, CNRS Éditions-Éditions de la MSH. \\ Meira, Sérgio et Franchetto, Bruna \\ 2005 «The Southern Cariban Languages and \\ the Cariban Family », International Journal \\ of American Linguistics 71(2) : 127-190.

\section{Melio, Maria Ignez Cruz} \\ 2005 lamurikuma: música, mito e ritual entre \\ os Wauja do Alto Xingu. Santa Catarina, \\ PPGAS-UFSC, thèse de doctorat. \\ Piedade, Acácio Tadeu de Camargo \\ 20040 Canto do Kawoka: música, cosmologia \\ e filosofia entre os Wauja do Alto Xingu. Santa \\ Catarina, PPGAS-UFSC, thèse de doctorat.

\section{Rouget, Gilbert} \\ 1980 La Musique et la Transe. Esquisse d'une \\ théorie générale des relations de musique et \\ de la possession. Paris, Gallimard.
}

\section{Résumé / Abstract}

Bruna Franchetto et Tommaso Montagnani, Flûtes des hommes, chants des femmes. Images et relations sonores chez les Kuikuro du Haut-Xingu - Cet article analyse les relations entre la musique du rituel des flûtes sacrées kagutu et les chants féminins tolo chez les Kuikuro du Haut-Xingu. Kagutu et tolo forment un système transrituel dans lequel une relation complexe entre hommes, femmes et esprits est établie au moyen de la musique et du langage. La musique kagutu est la voix des itseke, les esprits/animaux. Les flûtes kagutu, jouées par les hommes, reproduisent lors du rituel le son des noms des itseke : l'instrument est considéré comme pouvant imiter la voix des esprits et dire leurs noms, rendant ainsi leur présence sonore perceptible par les humains. Les chants féminins tolo sont basés sur les mélodies de kagutu. Selon les Kuikuro, les chants tolo «imitent » (ũ) les suites de flûte kagutu; il existe en réalité des différences importantes entre les deux répertoires, différences qui font l'objet de notre analyse.
Bruna Franchetto and Tommaso Montagnani, The flutes of men and the songs of women. Images and Acoustic Relations among the Kuikuro of the Upper Xingu - This article analyses the relationship between the ritual music of sacred flutes (kagutu) and women's songs (tolo) among the Kuikuro of the Upper Xingu. Together, kagutu and tolo form a transritual system, wherein music and language combine to create a complex relationship between men, women and spirits. Kagutu music corresponds to the voices of spirits/animals known as itseke, and when men play kagutu flutes during rituals, they reproduce the sound of the itseke's names. The instrument is thought to imitate the voices of spirits and to pronounce their names, thereby allowing humans to perceive their acoustic presence. Women's tolo songs are based on kagutu melodies. Accorrding to the Kuikuro, tolo songs "imitate" (ũ) kagutu flute suites; in fact, however, there are significant differences between the two repertoires and it is these differences that I explore in this paper. 